\title{
Future of the beam energy scan program at RHIC
}

\author{
Grazyna Odyniec $^{1 a}$ \\ ${ }^{1}$ Lawrence Berkeley National Laboratory, Berkeley, CA 94720, USA
}

\begin{abstract}
The first exploratory phase of a very successful Beam Energy Scan Program at RHIC was completed in 2014 with $\mathrm{Au}+\mathrm{Au}$ collisions at energies ranging from 7 to $39 \mathrm{GeV}$. Data sets taken earlier extended the upper limit of energy range to the $\sqrt{\mathrm{s}_{\mathrm{NN}}}$ of $200 \mathrm{GeV}$. This provided an initial look into the uncharted territory of the QCD phase diagram, which is considered to be the single most important graph of our field. The main results from BES phase I, although effected by large statistical errors (steeply increasing with decreasing energy), suggest that the highest potential for discovery of the QCD Critical Point lies bellow $\sqrt{\mathrm{s}_{\mathrm{NN}}} 20$ $\mathrm{GeV}$. Here, we discuss the plans and the preparation for phase II of the BES program, with an order of magnitude larger statistics, which is planned for 2018-2019. The BES II will focus on $\mathrm{Au}+\mathrm{Au}$ collisions at $\sqrt{\mathrm{s}_{\mathrm{NN}}}$ from 20 to $7 \mathrm{GeV}$ in collider mode, and from $\sqrt{\mathrm{s}_{\mathrm{NN}}} 7$ to $3.5 \mathrm{GeV}$ in the fixed target mode, which will be run concurrently with the collider mode operation.
\end{abstract}

\section{Introduction}

The RHIC (Relativistic Heavy Ion Collider) at Brookhaven National Laboratory, was designed and built to discover a Quark-Gluon Plasma (QGP). The top RHIC energy was specifically chosen to force the hot and dense system formed in ultra-relativistic nucleus-nucleus collisions through phase transition to the quark and gluon plasma phase. And, indeed, already the first results from RHIC demonstrate the formation of phase with partonic degrees of freedom [1]. The Quark-Gluon Plasma was discovered. However, the nature of the transition from hadronic degrees of freedom to the partonic ones, and back to the state of hadron gas, is totally unknown.

Theory gives very few insights into this problem. The generally accepted schematic representation of the QCD phase diagram is shown in Figure 1. Only the "edges" of this diagram are believed to be somehow understood: the lattice QCD calculations [2,3] predict a smooth cross-over transition to a plasma phase at the top RHIC energies, i.e. at critical temperature of 150-170 MeV and chemical potential $\mu_{\mathrm{B}} \sim 0$ (top left in Fig.1), while several QCD based calculations [4,5] show the first-order phase transition at lower $\mathrm{T}$ and higher $\mu_{\mathrm{B}}$ (right in Fig.1). The end of a first-order phase transition line marks the QCD Critical Point (CP), red circle in Fig.1. Although a position of the CP and the phase transition boundary are not known, there is enough information from lattice QCD to suggest that the most probable CP location would be somewhere between $\mu_{\mathrm{B}}=150$ and $500 \mathrm{MeV}$. This $\mu_{\mathrm{B}}$ interval corresponds to the energy range available in RHIC BES I program, allowing for the first time for the direct experimental search for $\mathrm{CP}$ and phase transition signatures.

\footnotetext{
a e-mail: G_Odyniec@lbl.gov
} 


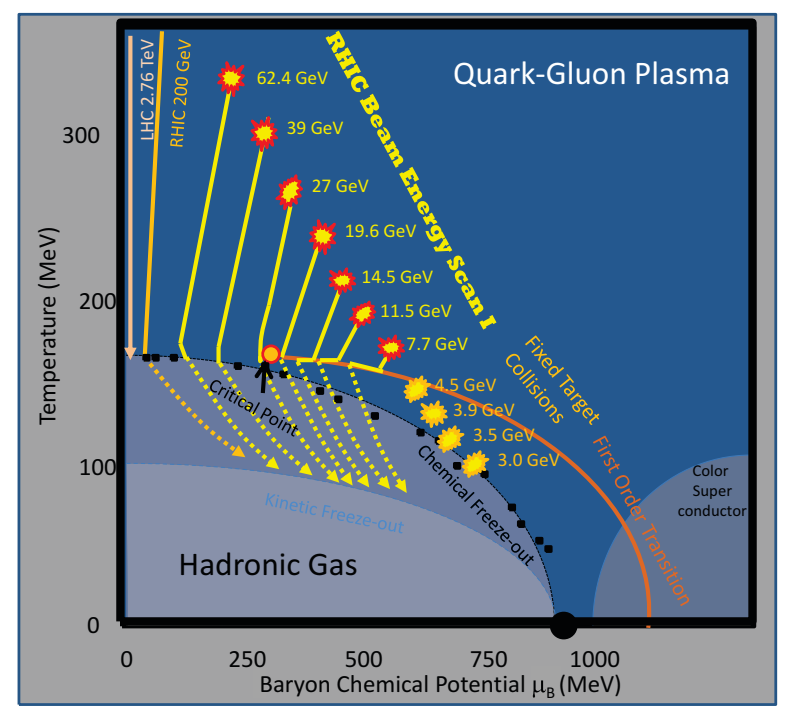

Figure 1: The picture of the QCD Phase Diagram with marked RHIC BES program coverage. Yellow trajectories represent schematics of the collision evolution at different energies at the BES program. The orange marked energies $(4.5,3.9,3.5$, and $3 \mathrm{GeV})$, to the right, display fixed-target program reach discussed in chapter 4 . See text for details.

\section{Results of BES phase I}

The BES program [6] goals are focused on three areas:

- The first one, and arguably the least complicated, is to scan the phase diagram with varying collision energy (what translates to changes in $\mu_{\mathrm{B}}$ and T) to find whether (and at what $\sqrt{\mathrm{s}_{\mathrm{NN}}}$ ) the key QGP signatures reported at the top RHIC energy are no longer observed. This could suggest that the system never entered the QGP phase remaining in the hadron gas phase throughout the entire collision process. The disappearance of a single signature would not be convincing evidence of the onset of deconfinement, because there might be some other phenomena not related to deconfinement which could be responsible for a similar effect, or our sensitivity to the particular observable could be reduced at lower energies. But, the modification or disappearance of several signatures simultaneously would constitute a more compelling case. The essential drivers of this part of the programs are: constituent quark number scaling, hadron suppression in central collisions characterized by $\mathrm{R}_{\mathrm{cp}}$, and correlations associated with the Chiral Magnetic Effect.

- The second goal is to find critical fluctuations, associated with a strong increase in the susceptibilities, which are expected in the vicinity of CP. However, because the finite size effects could wash out this critical behaviour, the third goal was proposed

- This third one is a search for evidence of the softening of the Equation Of State (EOS) as the system enters a mixed phase region and can be considered as an equivalent to the previous one (i.e. $\mathrm{CP}$ critical fluctuations search), as finding one will mean that the other one exists. Softening of EOS is implicitly associated with crossing a first-order phase transition. Promising observables in this search are: elliptic and direct flow of charged particles and of identified protons, net protons, and pions, azimuthally-sensitive femtoscopy, and fluctuations indicated by large jumps in baryon, charge and strangeness susceptibilities, as a function of system energy.

Figure 1 shows the reach of RHIC's BES program in the $\left(T, \mu_{B}\right)$ plane. The yellow lines represent possible reaction trajectories at energies $\sqrt{\mathrm{s}}_{\mathrm{NN}}=5,7.7,11.5,14.5,19.6,27$ and $39 \mathrm{GeV}$. All energies, with exception of 5 and $14.5 \mathrm{GeV}$, were run in 2010 and 2011. The $14.5 \mathrm{GeV}$ data ware obtained in 
2014 and completed the first phase of BES. The data taking at $\sqrt{\mathrm{S}_{\mathrm{NN}}}=5 \mathrm{GeV}$ was tested and found to be too challenging for the collider mode of RHIC operation. The orange numbers to the right of the Fig.1 show the energies for the fixed-target program planed for phase II of the BES program, discussed in chapter 4 . This choice of energies provided almost uniform coverage of the unknown ( $T$, $\left.\mu_{B}\right)$ territory and allowed us to narrow down an area of interest for BES phase II studies.

Phase I of the BES program allowed STAR to extend the $\mu_{\mathrm{B}}$ reach of RHIC from a few tens of $\mathrm{MeV}$ at $\sqrt{\mathrm{s}_{\mathrm{NN}}}=200 \mathrm{GeV}$ up to approximately $400 \mathrm{MeV}$ at $\sqrt{\mathrm{s}_{\mathrm{NN}}}=7.7 \mathrm{GeV}$. The performance of both the collider and the experiments were excellent throughout the entire energy range explored to date.

The BES Phase I analyses results, although still considered preliminary, already have allowed STAR to close-in on some of the goals outlined above. Moreover, they specify a clear path for the BES phase II, which should lead to conclusions regarding all of the BES goals.
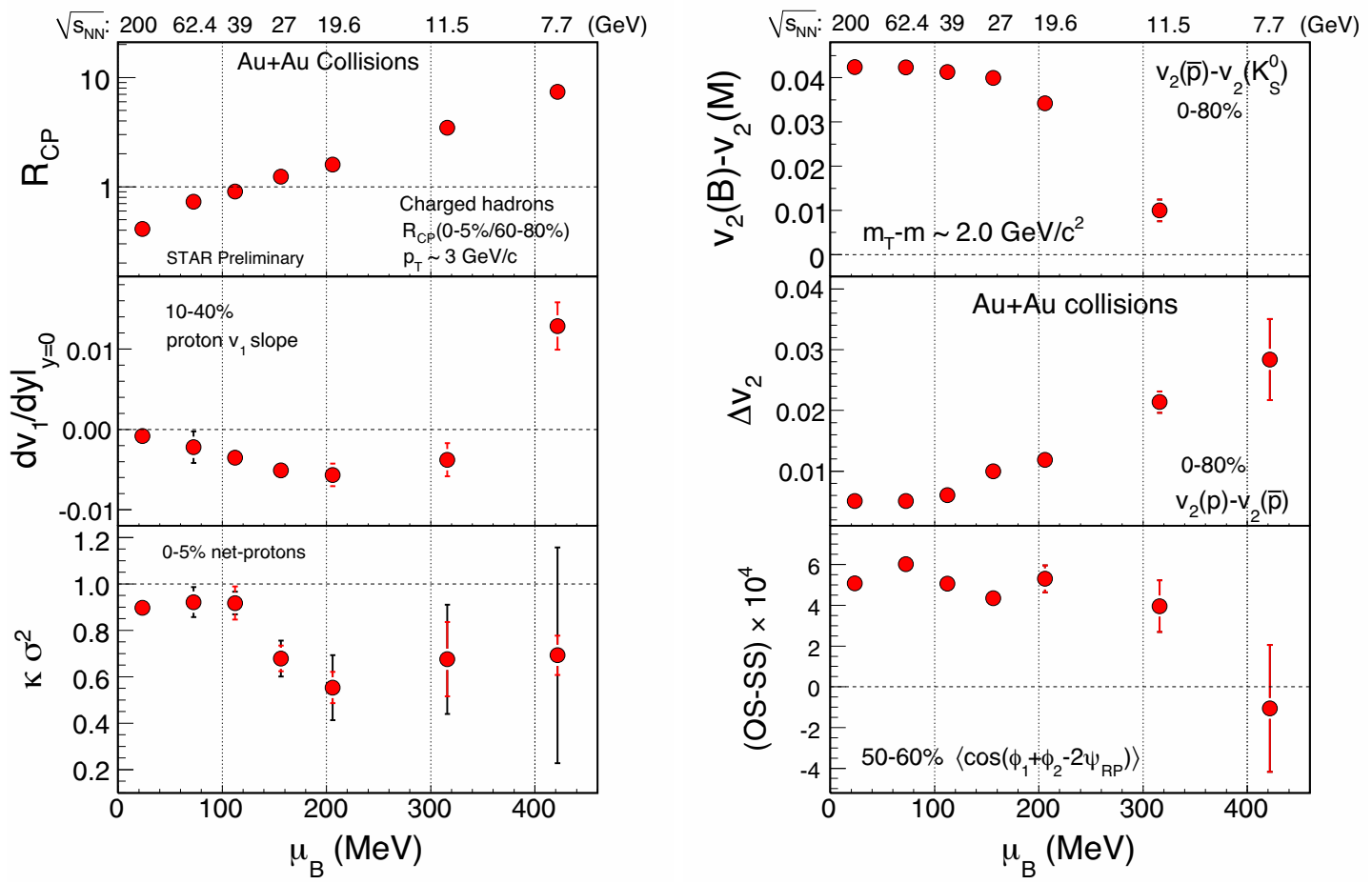

Figure 2: A summary of selected results from $\mathrm{Au}+\mathrm{Au}$ collisions in the BES-I program as a function of baryon chemical potential. Left panel (from the top): mid-rapidity results for the nuclear modification factor for charged hadrons, the slope of direct flow of protons for $10-40 \%$ centrality, $\kappa \sigma^{2}$ for net-protons for $0-5 \%$ centrality. Right panel (from the top): the difference in baryon and meson $\mathrm{v}_{2}$ at $\mathrm{m}_{\mathrm{T}}-\mathrm{m}=2 \mathrm{GeV} / \mathrm{c}^{2}$ for minimum bias collisions, the difference between $\mathrm{v}_{2}$ of protons and antiprotons for minimum bias collisions, and the difference between opposite-sign and same-sign charge correlations with respect to the reaction plane for $50-60 \%$ centrality.

A summary of the most intriguing results from BES I analysis is presented in Figure 2. The more detailed description of the analysis and its findings can be found in these proceedings [7].

All observables, as seen in Fig.2, exhibit interesting trends as a function of changing $\mu_{\mathrm{B}}$, as the beam energy increases from 7.7 to $200 \mathrm{GeV}$. Note, that the connection to $\mu_{\mathrm{B}}$ is not exact, as the $\mu_{\mathrm{B}}$ assignment at a given collision energy is dependent on the centrality, and the various analyses have different centrality selection.

The left upper panel of Fig. 2 shows the decrease of the charged particles nuclear modification factor, $\mathrm{R}_{\mathrm{cp}}$, in $\mathrm{Au}+\mathrm{Au}$ central ( $5 \%$ most central) collisions as a function of $\mu_{\mathrm{B}}$. The $\mathrm{R}_{\mathrm{cp}}$ crosses the 
value of one around $\mu_{\mathrm{B}}=155 \mathrm{MeV}$, corresponding to $\sqrt{\mathrm{S}_{\mathrm{NN}}}=27 \mathrm{GeV}$, suggesting that below this energy partonic energy loss may be very small or not present at all. The middle left plot show the nonmonotonic behavior of slope of the directed flow of protons $\left(\mathrm{dv}_{1} / \mathrm{dy}\right)$ in mid-central collisions $(10-40$ $\%$ centrality) with a clear minimum somewhere above $\mu_{\mathrm{B}}=205 \mathrm{MeV}$ (below $\sqrt{\mathrm{s}_{\mathrm{NN}}}=19.6 \mathrm{GeV}$ ). This observable, which is driven by the pressure gradient developed in the system, is sensitive to possible first-order phase transition effects. The lower left panel shows the variation of the $\kappa \sigma^{2}$ of the netproton number distribution with $\mu_{\mathrm{B}}\left(\sim \sqrt{\mathrm{S}_{\mathrm{NN}}}\right)$. The present statistics is not sufficient to clarify whether $\kappa \sigma^{2}$ monotonically decreases with $\mu_{\mathrm{B}}$ or there is a minimum between $\mu_{\mathrm{B}}=155 \mathrm{MeV}$ and $315 \mathrm{MeV}$ $\left(\sqrt{\mathrm{s}_{\mathrm{NN}}}=27\right.$ to $\left.11.5 \mathrm{GeV}\right)$ as observed for the proton $\mathrm{dv}_{1} /$ dy. The right top panel of Fig.2 shows that the difference in the $\mathrm{v}_{2}$ of baryons and mesons in minimum-bias $\mathrm{Au}+\mathrm{Au}$ collisions at $\mathrm{m}_{\mathrm{T}}-\mathrm{m} \sim 2$ $\mathrm{GeV} / \mathrm{c}^{2}$ decreases with increasing $\mu_{\mathrm{B}}$ (decrease in $\left.\sqrt{\mathrm{S}_{\mathrm{NN}}}\right)$. This difference starts to decrease after $\mu_{\mathrm{B}}=$ $155 \mathrm{MeV}\left(\sqrt{\mathrm{S}_{\mathrm{NN}}}=27 \mathrm{GeV}\right)$. The existence of a difference between the $\mathrm{v}_{2}$ of baryons and mesons at intermediate $\mathrm{p}_{\mathrm{T}}$ is the key to the experimental observation of NCQ scaling and the observation of the partonic collectivity at top RHIC energy. The middle panel shows the difference in $\mathrm{v}_{2}$ between baryons and antibaryons (shown for protons and antiprotons) which starts to increase with $\mu_{\mathrm{B}}$ (decreases with $\sqrt{\mathrm{s}_{\mathrm{NN}}}$ ). Comparison to models suggests that these observations are consistent with the dominance of the hadronic scenario in the investigated systems at lower energies. The lowest right plot shows the difference in the observed signal of dynamical charged correlations between same-sign and opposite-sign charges for $50-60 \%$ central $\mathrm{Au}+\mathrm{Au}$ collisions as a function of $\mu_{\mathrm{B}}\left(\sqrt{\mathrm{S}_{\mathrm{NN}}}\right)$. One possible explanation of this effect at the top of RHIC energies is the Chiral Magnetic Effect, which implies the formation of a deconfined state of quarks and gluons with chiral symmetry restored. The observed trend suggests the vanishing and finally the absence of this state at the lowest energies ( $\left.\sqrt{\mathrm{s}_{\mathrm{NN}}}=7.7 \mathrm{GeV}\right)$.

All these obsevables are hurt by the large statistical errors, which make it impossible to draw strong conclusions. Several demonstrate the dominance of parton regime at the BS high energies, these signatures either disappear, lose significance, or lose sufficient reach in the low energy region of the scan. Note, that for example, hard probes become less accessible at lowest collision energies. We observed also some indication of a softening of EOS around 11.5-19.6 GeV that could be indicative of a $1^{\text {st }}$ order phase transition, as well as a suggestive signs of fluctuations ( $\kappa \sigma^{2}$ analysis), which could be a signal of anticipated critical fluctuations, but these are highly statistics hungry analyses and conclusions need to be postponed to the analysis of larger statistics of BES II.

\section{BES phase II}

The statistics collected during BES Phase I are not sufficient to reach a confident conclusion on the three goals explained in the previous chapter. Therefore, the STAR Collaboration proposes the BES phase II program of precision measurements to map out the QCD phase diagram with an order of magnitude increase in data samples. The BES phase II is planned to take data in 2018-2019. It will focus on a narrow energy window, where signatures of QGP seem to change or even disappear, i.e. below $\sqrt{\mathrm{S}_{\mathrm{NN}}} 20 \mathrm{GeV}$.

Additionally, there is a plan to run STAR in fixed-target mode concurrently with collider mode during BES Phase II. This would allow the extension of the range of accessible baryon chemical potential from the current maximum of just above $\mu_{\mathrm{B}} \sim 400 \mathrm{MeV}$ up to $\mu_{\mathrm{B}} \sim 750-800 \mathrm{MeV}$, corresponding to a collision energy $\sqrt{\mathrm{s}}_{\mathrm{NN}} \sim 3 \mathrm{GeV}$.

\subsection{High Luminosity for BES II at RHIC}

The major trust of the BES Phase II program is to make high statistics measurements of $\mathrm{Au}+\mathrm{Au}$ collisions at $\sqrt{\mathrm{S}_{\mathrm{NN}}}<20 \mathrm{Gev}$. This requires the RHIC operations with luminosity significantly higher than those achieved during BES I. This will be accomplished by constructing an electron cooling system to reduce the transverse beam emittance and by developing the ability of stretching the bunches longitudinally in order to reduce the intra-beam Coulomb scattering. 
RHIC was designed and optimized for the luminosity at ${ }_{\mathrm{s}_{\mathrm{NN}}}=200 \mathrm{GeV}$. At lower energies, the beam is not as well-focused and the collisions rate is low. The above mentioned electron cooling will provide the adequate intensity for BES Phase II. According to simulations, with the proposed electron superconducting RF gun, the luminosity will increase by a factor of $2-5$ at $\sqrt{\mathrm{s}_{\mathrm{NN}}}=7.7 \mathrm{GeV}$ and by a factor of 8-20 at $\sqrt{\mathrm{S}_{\mathrm{NN}}}=20 \mathrm{GeV}$. Additional luminosity increase will be made possible by stretching the beam bunches beyond the usual 6 ns length. Figure 3 shows the projection of average store luminosity for Au ions in RHIC. The lines represent the most "optimistic" (red) and the "pessimistic" (blue) scenarios with electron cooling. The open squares show the luminosities of the BES I run.

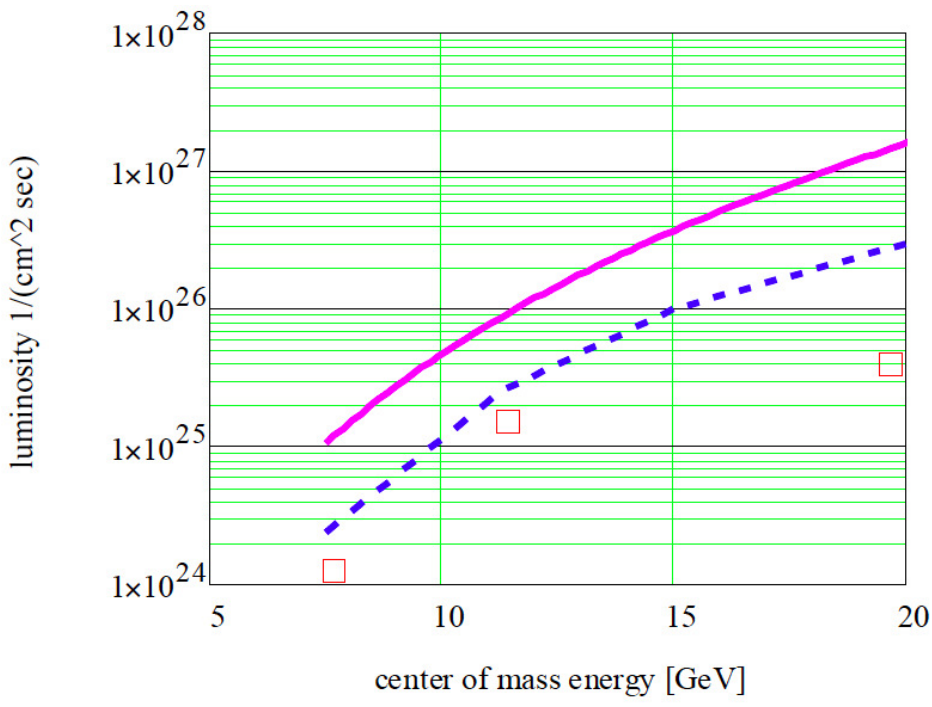

Figure 3: Projection of average store luminosity for Au ions in RHIC. The red line represents the most optimistic estimates for the luminosity with electron cooling implemented, while the blue one shows the pessimistic scenario. The open squares are the luminosities reached in BES I.

All of these advances will allow achieving the event statistics required for the key measurements of the BES II program.

\subsection{Detector upgrades}

Parallel with studies on RHIC luminosity increase, the intensive work of getting experiments ready for the challenges of the BES II program is in progress. The STAR experiment will benefit from a number of improvements to various sub-systems. The most important are described below.

\subsubsection{Inner Sector TPC upgrade (iTPC)}

The main detector of the STAR experiment, the STAR TPC, will be upgraded to increase segmentation of the inner sector's pad planes and to renew the inner sector wires, which are showing signs of aging.

Unlike the outer TPC sectors, the current inner TPC pad row geometry does not provide hermetic coverage at all radii. About only $20 \%$ of the path length of a charged particles path traversing an inner sector of the TPC is sampled by the current pad plane and electronics readout. With the proposed upgrade, the path length coverage in the inner sectors will be increased to $100 \%$.

This upgrade will provide better momentum resolution, better $\mathrm{dE} / \mathrm{dx}$ resolution, higher track reconstruction efficiency, and most importantly it will increase acceptance at high rapidity to $-1.7<\eta$ $<1.7$, to be compared with the current TPC configuration of $-1<\eta<1$. Figure 4 shows the improved acceptance in $\mathrm{p}_{\mathrm{t}}$ and $\mathrm{y}$ for reconstructed pions, kaons and protons. Although iTPC will allow 
improved tracking and acceptance $\left[\left(y, p_{t}\right)\right.$ coverage] through the entire phase space, the most significant change is observed in simulations of forward/backward direction: $1<|\eta|<1.5$. The blue points in Figure 4 represent the coverage of the original TPC, the red ones the one for the iTPC.

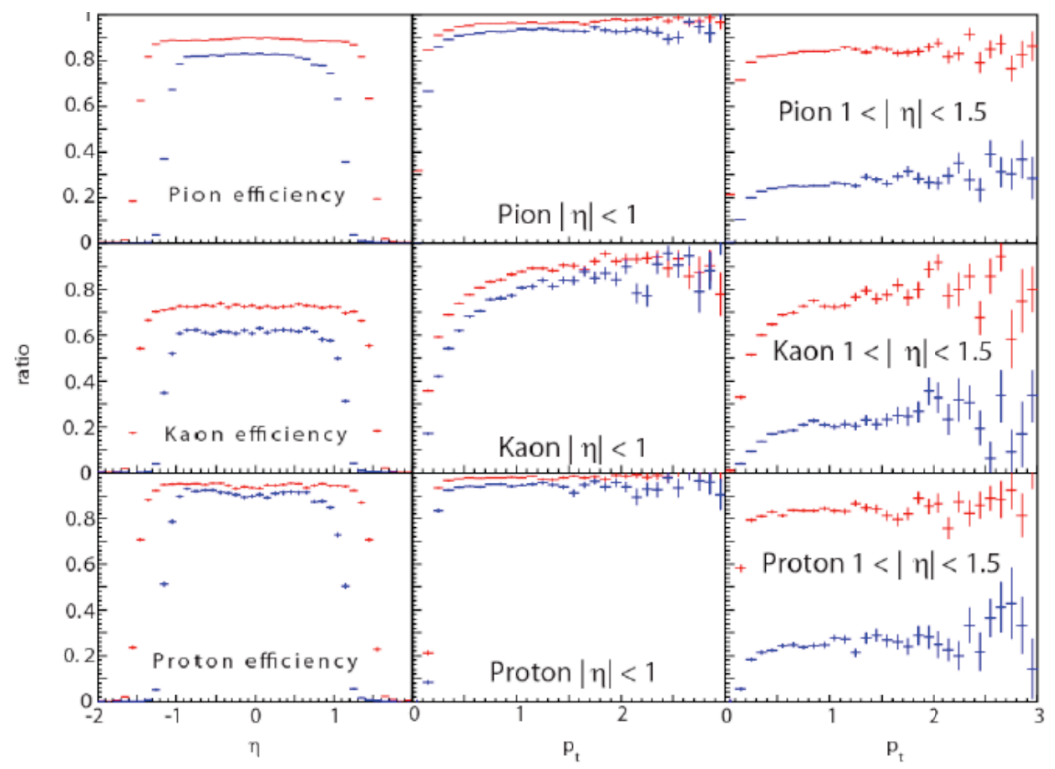

Figure 4. Pseudorapidity and transverse momentum dependence of the detection efficiencies for pions (top panel), kaons (middle panel), and protons (bottom panel). Blue and red points are the results for TPC and iTPC, respectively.

The new measurement capabilities of STAR after the iTPC upgrade are a vital part of the new BES II effort. The iTPC will allow us to study rapidity dependence of freeze-out dynamics, it will extend measurements of $\mathrm{v}_{1}$ beyond the information contained in the slope of $\mathrm{v}_{1}(\mathrm{y})$ close to midrapidity (a broader rapidity acceptance will expand our understanding of the role of baryon transport on the $\mathrm{v}_{1}$ measurements), it will allow study of the rapidity dependence of higher moments of net-proton/netcharge distribution to understand the role of charge/baryon number conservation, and will improve many more observables. It will also improve the low $\mathrm{p}_{\mathrm{t}}$ acceptance by reducing systematic uncertainties associated with extracting particle yields.

\subsubsection{Event Plane Detector (EPD)}

The second most important improvement to the STAR detector experimental set-up is an addition of a newly built dedicated event-plane and centrality detector, EPD, which will be placed in the forward rapidity region $2<|\eta|<4$. With segmentation in both radial and azimuthal directions, the detector will provide precise measurements of both the event plane and the collision centrality. Measurements of elliptic flow in BES I used the TPC for the event plane determination. Non-flow effects have been reduced by keeping an $\eta$ gap (currently $\sim 0.1-0.2$ ) between the particles used for the correlation measurements and the event plane measurement. An EPD centered at a pseudorapidy of 3 units would result in an $\eta$ gap of about 2 units of pseudorapidity, decreasing the non-flow effects significantly. An EPD would also improve significantly physics performance and facilitate physics interpretation of the direct flow measurements (in direct flow analysis in BES I the BBC detectors were used for the event plane determination, which have rather modest event-plane resolution). 


\subsubsection{End-Cap Time-Of-Flight (eTOF)}

Installation of the End-Cap TOF will enable particle identification in the forward direction. One prototype sector of eTOF will be included in the BES II run. Although the eTOF will have a narrow azimuthal acceptance, this will enable key inclusive measurements.

The enhanced performance made possible by the iTPC, EPD and eTOF will not only benefit the BES Phase II physics program, but will also be crucial for STAR's future program with $p+p / p+A$ and $\mathrm{e}+\mathrm{p} / \mathrm{e}+\mathrm{A}$ collisions at forward higher-rapidity regions.

\subsection{Trigger for BES II}

The transverse beam size at the lowest RHIC energies was significantly greater than at $\sqrt{\mathrm{S}_{\mathrm{NN}}}=200$ $\mathrm{GeV}$, causing collisions of ions in the beam halo with either the beam pipe or support-structure materials. At $\sqrt{\mathrm{s}_{\mathrm{NN}}}=7.7 \mathrm{GeV}, 80-98 \%$ of the triggered reactions came from such beam on beam-pipe collisions. The situation will improve with installation of an RF electron cooling system. A total increase in luminosity of about a factor 10 is expected, which will result in a trigger rate of several $\mathrm{kHz}$ at the highest BES energy. To exploit this, it is essential to trigger on all good $\mathrm{Au}+\mathrm{Au}$ collisions with a reconstructable vertex.

\section{Fixed target program}

Intriguing threshold behaviour was observed in BES I data at the lowest energy of $\sqrt{\mathrm{s}_{\mathrm{NN}}}=7.7 \mathrm{GeV}$, see Fig. 5. Similar observations were also made by the NA49 Collaboration at CERN SPS [8].
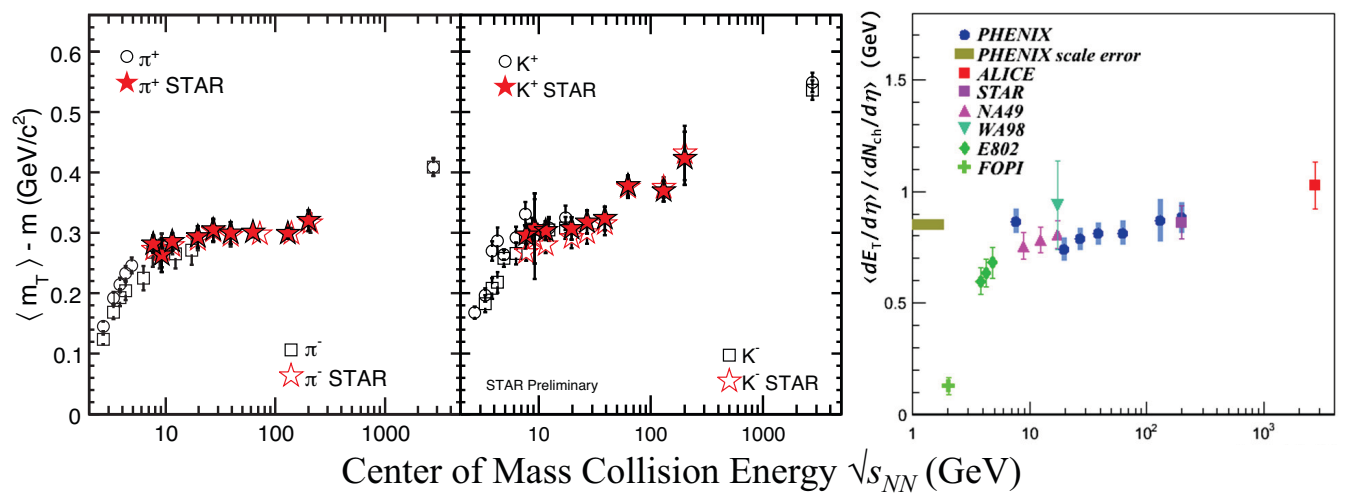

Figure 5: Left panel: Center-of-mass energy dependence of $\left\langle\mathrm{m}_{\mathrm{T}}>-\mathrm{m}\right.$ of $\pi$ and $\mathrm{K}$ in central Au+Au collisions at RHIC. Also shown are corresponding results from AGS [9, 10, 11, 12, 13, 14], SPS [15, 16] and LHC [17]. Right panel: The average transverse energy, scaled by the charge particle multiplicity at mid-rapidity, as a function of collision energy observed by PHENIX, STAR, ALICE, NA49, WA 98, E802 and FOPI Collaborations [18].

These observations led to the suggestion that this energy might correspond to the onset of deconfinement. STAR will test this hypothesis with the fixed target mode as the lowest energies (below $7.7 \mathrm{GeV}$ ) have proven to be challenging for the collider. It has been demonstrated that with the addition of a stationary gold target placed inside the beam pipe at the entrance to the STAR TPC, energies of $\sqrt{\mathrm{s}_{\mathrm{NN}}}=3.0,3.2,3.5,3.9$, and $4.5 \mathrm{GeV}$ could be studied, what will provide an extension of the BES II program down to beam energies significantly below the lower limit of RHIC operations in 
the collider mode (see Fig.1). These energies correspond to collisions between projectiles tuned for collisions at 7.7, 9.1, 11.5, 14.5 and $19.6 \mathrm{GeV}$ respectively and the stationary gold fixed-target. Fixedtarget collisions would allow for extension of the range of accessible baryon chemical potential from the current maximum of about $\mu_{\mathrm{B}} \sim 400 \mathrm{MeV}$ up to $\sim 750-800 \mathrm{MeV}$ in the QCD phase diagram. The proof of principle was already successfully tested in run 14 during the $14.5 \mathrm{GeV} \mathrm{Au}+\mathrm{Au}$ program. The stationary gold target was mounted at the entry to the TPC as shown in Fig. 6. Preliminary data show that the fixed-target trigger was properly selecting fixed-target events and rejecting beam-beam collisions. Figure 7 shows a display of one of the fixed-target events from run 14.

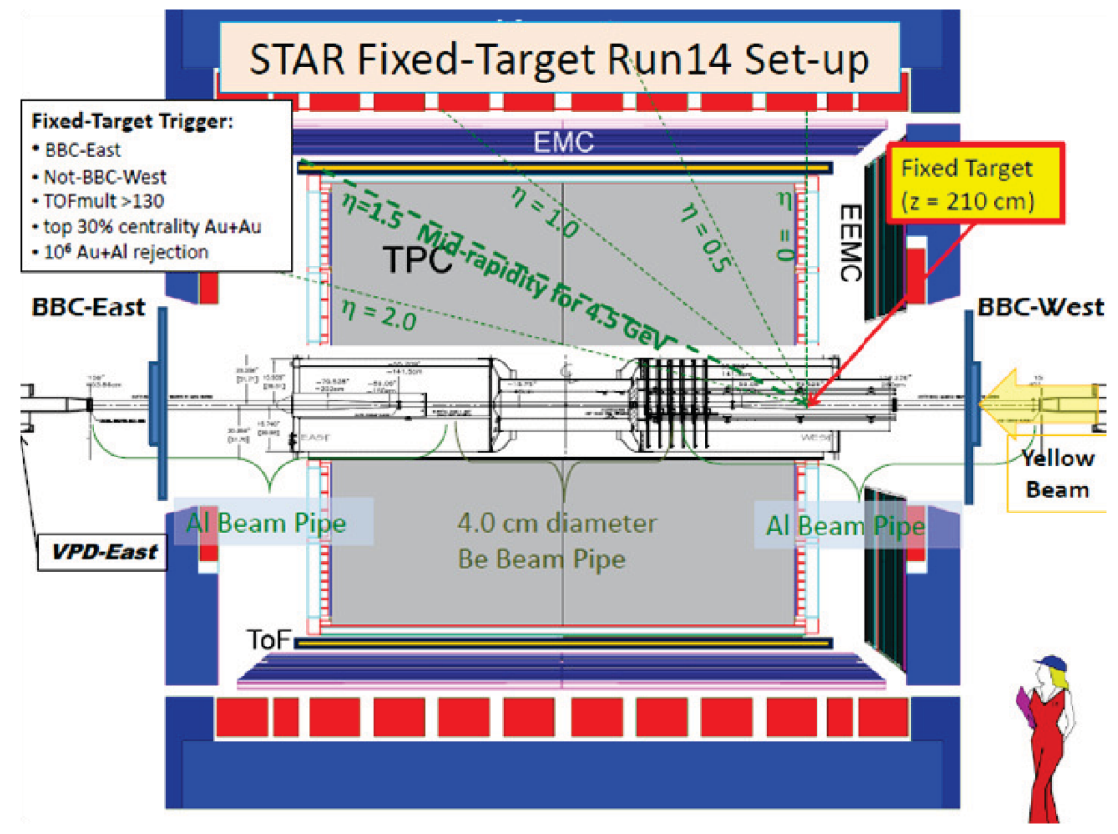

Figure 6: A schematic diagram of STAR showing the fixed-target location for the test in 2014. The target is a $1 \mathrm{~mm}$ thick gold foil, the projectiles are ions from the halo of the yellow beam (west side).

It will be possible to cross-check the results of the fixed-target program with those of the collider program. This very important check can be done by tuning the collider for $62.4 \mathrm{GeV}$ collisions and take fixed-target data with one of the beams on the stationary target. These fixed-target datasets will be taken at $\sqrt{\mathrm{s}_{\mathrm{NN}}}=7.7 \mathrm{GeV}$, and could be directly compared to the $7.7 \mathrm{GeV}$ collider data.

The primary physics goals of this part of the program will focus on observables, which are sensitive to a softening of the equation of state, as it is expected that the first entry into the mixedphase will occur at energies below the onset of deconfinement [19, 20, 21]. 


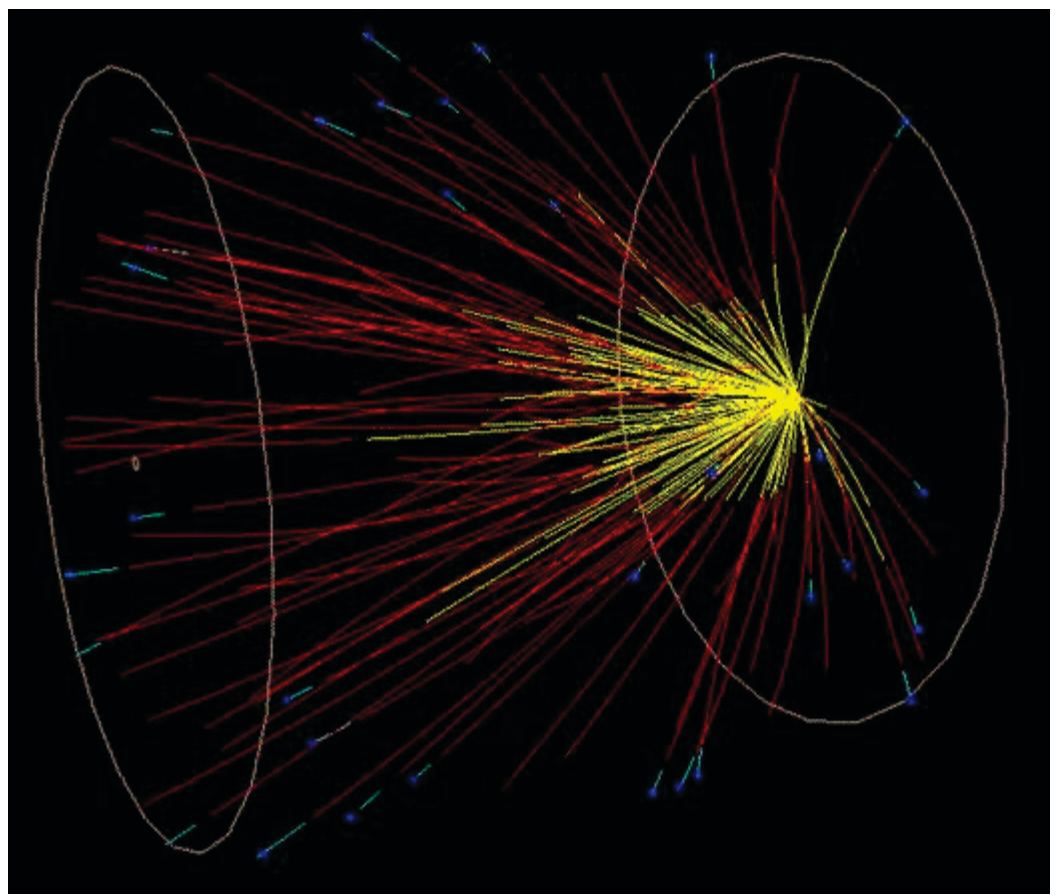

Figure 7: The reconstructed tracks from an interaction between a nucleus in the beam halo and a gold nucleus in the stationary target positioned at the entrance to the TPC $(\mathrm{z}=210 \mathrm{~cm})$, see figure 6 . This is a central event at ${\sqrt{\mathrm{s}_{\mathrm{NN}}}}$ $=3.9 \mathrm{GeV}$ (Au beams with $\mathrm{E}_{\text {Total }}$ of $7.25 \mathrm{GeV}$ on stationary target).

\section{Concluding remarks}

RHIC "sits" at a "sweet spot" in energy, in which rapid changes occur in a number of signatures for energies up to approximately $20 \mathrm{GeV}$, while remaining surprisingly stable over two orders of magnitude to the LHC. The BES I data shows that several observables associated with the formation of partonic phase at the top RHIC energies have been turned-off at lower energies and that there, at the lower energies, hadronic interactions dominate the scene. An important measurement towards establishing a position of the critical point and the location of the $1^{\text {st }}$ order phase transition has been made. Those results provided clear directions for the second part of the BES program which will cover energies below $\sqrt{\mathrm{s}_{\mathrm{NN}}}=20 \mathrm{GeV}$. Collider mode operation will span from $\sqrt{\mathrm{s}_{\mathrm{NN}}}$ of 20 to $7.7 \mathrm{GeV}$, while fixed-target will extend a reach to energies as low as $\sqrt{ }_{\mathrm{NN}_{\mathrm{N}}}=\sim 3 \mathrm{GeV}$. Both collider and experiment are prepared for a challenge. With BES phase II the most exciting physics is still to come.

\section{References}

1. STAR White Paper, J. Adams et al., [STAR Coll.], Nucl. Phys. A 757 (2005) 102; J. Adams et al., [STAR Coll.], Phys. Rev. Lett. 92 (2004) 052302; Phys. Rev. Lett. 95 (2005) 122301

2. F. Karsch et al., Nucl. Phys. Proc. Suppl. 129 (2004) 614

3. M. Cheng et al., Phys. Rev. D 79, 074505 (2009) and references therein

4. Y. Aoki et al., Nature 443, 675 (2006)

5. S. Ejiri, Phys. Rev. D 78, 074507 (208); E.S. Bowman and J.I.Kapusta, Phys. Rev. C 70, 015202 (2009)

6. BES STAR White Paper, M.M.Aggarwal et al., [STAR Coll.], arXiv:12007.2613 
7. D. McDonald, this proceedings

8. C.Alt et al., [NA 49 Collaboration], Phys. Rev. C 81 (2010) 024911

9. L. Ahle et al., [E866 and E917 Coll.], Phys. Lett. B 476 (2000) 1

10. L. Ahle et al., [E866 and E917 Coll.], Phys. Lett. B 790 (2000) 53

11. L. Ahle et al., [E802 Coll.], Phys. Rev. C 57 (1998) 466

12. L. Ahle et al., [E802 and E866 Coll.], Phys. Rev. C 60 (1999) 044904

13. J.L.Klay et al., [E895 Coll.], Phys. Rev. Lett. 88 (2002) 102301

14. J.Berrette et al., [E877 Coll.], Phys. Rev. C 62 (2000) 024901

15. S.V.Afanasiev, et al., [NA49 Coll.], Phys. Rev. C 66 (2002) 054902

16. C.Alt et al., [NA49 Coll.], Phys. Rev. C 77 (2008) 024903

17. E.O'Brien et al., [PHENIX Coll.], Nucl. Phys. A 904, 905 (2013], 264c

18. B.Abelev et al., [ALICE Coll.], Phys. Rev. C 88 (20130 044910

19. I.C.Arsene et al., Phys. Rev. C 75 (2007) 034902

20. J.Randrup, Phys. Rev. C 79 (2009) 054911

21. J.Randrup, Phys. Rev. C 82 (2010) 034902 